\title{
INTRUSION FLOW INTO A DENSITY STRATIFIED RESERVOIR
}

\author{
HANAN ABDULMOTALAB M. OMAR
}

(Received 20 March 2017; first published online 5 July 2017)

\begin{abstract}
2010 Mathematics subject classification: primary 76B07.
Keywords and phrases: intrusion flow, stratified fluid, potential flow.
\end{abstract}

Steady two-dimensional flow from an angled structure into a lake or a reservoir where the interface between the intrusion and the ambient fluid separates from a solid wall is considered. This work is of importance for understanding reservoir dynamics so that water quality can be maintained. The fluid is assumed to be of finite depth and the incoming channel makes an angle $\alpha$ with the horizontal axis. The problem is formulated using conformal mapping and integral equation techniques and the resulting problem is solved using a surface angle approach. The shape of the interface is computed for a range of entry angles and flow rates. Exact solutions are presented at a high flow rate and compared with the solutions to the nonlinear problem. These results have appeared in [1]. Solutions with waves are shown to exist on the interface at small flow rate and these are computed at very small entry angles using a physical plane method. The case in which the lake or the reservoir is stratified in density is also considered and the separation height is determined for different values of the stratification. These results have appeared in [2]. In all cases, the parameter space in which steady solutions exist is studied and limiting solutions are obtained. The results have implications for the design of efficient inflow structures for reservoirs and for water quality management.

\section{References}

[1] H. A. Omar and G. C. Hocking, 'Dense underflow into a lake or reservoir - supercritical flow solutions', Appl. Math. Model. 42 (2017), 449-461.

[2] H. A. Omar and G. C. Hocking, 'Dense underflow into a lake or reservoir - sub-critical flow', Appl. Math. Model. 46 (2017), 465-475.

Thesis submitted to Murdoch University in June 2015; degree awarded on 21 October 2016; supervisors Graeme Hocking and Duncan Farrow.

(c) 2017 Australian Mathematical Publishing Association Inc. 0004-9727/2017 \$16.00 
HANAN ABDULMOTALAB M. OMAR, Department of Mathematics and Statistics, Murdoch University, WA 6150, Australia e-mail: hananomar42@yahoo.com 